\title{
Neuropsychological Function and REM Sleep in Schizophrenic Patients
}

\author{
Stephan F. Taylor, Robert S. Goldman, Rajiv Tandon, and \\ James E. Shipley
}

To test the hypothesis that rapid eye movement (REM) sleep in schizophrenic patients is associated with cogritive function, we studied 18 schizophrenic inpatients by means of electroencephalograms taken during sleep in their own hospital beds after a minimum 2-wk medication withdrawal period. Patients underwent neuropsychological tests to measure memory function and other aspects of cognitive performance. REM sleep measures demonstrated positive and negative correlations with cognition and memory measures, depending on when REM occurred after sleep onset. Minutes of REM sleep and REM density in the first period correlated negatively with performance, while REM minutes occurring after the first REM period correlated positively with neuropsychological performance. Further work should test whether phasic REM sleep regulation at the beginning of the night plays a compensatory role for neuropsychological dysfunction in schizophrenics.

Attempts to understand rapid eye movement (REM) sleep have focused on its hypothesized role in memory and learning processes. Animal studies suggest that REM sleep may play a role in consolidating recently acquired material in long-term storage (Horne and McGrath 1984; Smith 1985). Studies in humans using REM deprivation have yielded some support for the memory-consolidation hypothesis but are difficult to interpret, in part because of the disruption of sleep caused by REM deprivation (Fowler et al 1973; Greenberg et al 1983). Correlational studies between performance and REM measures in humans have demonstrated a direct relationship between REM and recent learning for complex and emotionally significant material (Dujardin et a 1990). Several studies in older normal subjects suggest a broader connection between REM and intellectual function, demonstrating a positive correlation of REM time with IQ and memory measures such is the Wechsler Memory Scale (WMS) (Feinberg et al 1967; Kahn and Fisher 1969; Prinz 1977).

Studies of individuals with central nervous system disturbances have demonstrated an association for REM sleep with memory and general intellectual function. Abstinent alcoholics demonstrated a positive correlation for digit span and arithmetic problem-

From the Schizophrenia Program and the Sleep Diagnostic and Re zearch Program, University of Michigan, Department of Psychiatry

Address reprint requests to Stephan F. Taylor, University of Michigan, Department of Psychiatry, University Hospital $9 \mathrm{C}$ Box 0120,1500 E. Medical Center Dr., Ann Arbor, MI 48109.

Received March 16, 1992; revised May 12, 1992. 
solving with time spent in REM sleep (Benson et al 1978). Feinberg et al (1967) reported positive correlations between WMS scores and REM latency, and between performance IQ and REM time in demented patients. Studies of developmentally disabled individuals have found positive correlations for full-scale IQ with REM time and REM activity (Feinberg 1968; Castaldo and Krynicki 1974). These correlational studies, which examine abnormal individuals with a broader range of intellectual performance than that found in normal subjects, suggest that subjects who perform better on tests of memory and cognition spend more time in REM sleep.

We found no studies measuring intellectual function and possible connections wih REM sleep in schizophrenic patients. Given the well-documented cognitive deficits in schizophrenia (Levin et al 1989; Taylor and Abrams 1984; Saykin et al 1991), schizophrenic patients may bear some resemblance to the globally impaired groups studied by Feinberg and other investigators. Further studies may provide an opportunity to extend these previous observations.

We performed a study to test the specific hypothesis that memory and cognitive performance were positively correlated with REM time in schizophrenic patients. We confirmed this positive correlation with REM time occurring after the first REM period, but we also found a negative association for memory and cognitive performance with REM time and density in the first REM period.

\section{Methods}

\section{Subjects}

The study population consisted of 18 patients ( 6 women, 12 men) with an average age of $27.3 \mathrm{yr}(\mathrm{SD}=7.3$, range $=19-46 \mathrm{yr})$, an average education of $13.4 \mathrm{yr}(\mathrm{SD}=2.8$, range $=9-18)$, and an average illness duration of 6.5 yr $(S D=6.8$, range $=0.7-23$ yr). All patients gave informed consent to participate in studies in the Schizophrenia Program at the University of Michigan. An experienced research nurse administered the Schedule for Affective Disorders and Schizophrenia (SADS; Endicott et al 1978). All patients met both DSM-III-R (American Psychiatric Association 1987) and RDC (Spitzer et al 1978) criteria for schizophrenia. Patients who had been on neuroleptic medication did not receive any psychotropic drugs for a minimum of two weeks before the first night of sleep recordings. Eight of the patients had not had previous neuroleptic exposure. Patients who had a history of drug or alcohol abuse within the past six months or significant medical or neurological problems, including historical or clinical evidence of narcolepsy, were excluded from the study. The patients underwent clinical ratings by trained raters, blind to sleep electroencephalographic (EEG) or neuropsychological findings, by means of the 18-item Brief Psychiatric Rating Scale (BPRS; Overall and Gorham 1962) and the Scale for the Assessment of Negative symptoms (SANS) (Andreasen 1983).

\section{Sleep Electroencephalographic Study}

On the days when the patients underwent sleep studies, daytime napping was not permitted. Polysomnography (PSG) was performed while the patients slept in their own hospital beds. Data was transmitted via a multiplexing system (Telefactor Corp., West Conshohocken, PA) to a nearby sleep laboratory control room and recorded on a Grass 
Model 78B polygraph, yielding band-pass settings of $0.5-30 \mathrm{~Hz}$. The first night of recording involved a full-montage PSG, including EEG (C3/A2), electrooculogram (EOG), submental electromyogram (EMG), respiratory monitoring (nasal-oral thermistors, abdominal and chest strain gauges, and ear oximetry), and electrocardiogram (ECG) and EMG of the anterior tibialis muscle (to assess periodic leg movements). The first night was used both to exclude any primary sleep disorder and to acclimate the subjects to the recording conditions. Data from the second night of PSG recording, utilizing EEG, EOG, and EMG for sleep-staging, provided PSG measures for the analysis.

Trained raters, who were blind to diagnosis and neuropsychological testing results, scored the sleep records according to modified Rechtschaffen-Kales criteria (Rechtschaffen and Kales 1968). REM latency was defined as the time from sleep onset until the onset of the first REM period, 3 min or more in duration, minus intervening time awake. Sleep onset was scored as the time between lights out and the first $10 \mathrm{~min}$ of stage 2, 3, or 4 sleep that was not interrupted by more than 2 min of stage 1 sleep or 1 min of stage 1 plus 1 min of waking. Other REM measures included REM activity (sum of visually scored eye-movement density from each $60-\mathrm{sec}$ REM epoch on a scale of 0-8) and REM density (REM activity/REM time).

\section{Neuropsychological Tests}

Subjects underwent a battery of neuropsychological tests, usually within two days of the sleep study (mean $=1.4$ days); none received psychotropic medication during test days. Testing and scoring were performed by raters who had no knowledge of the results of the sleep studies. We selected three tests of memory. In the Bushke Selective Reminding Task (SRT) (Buschke and Fuld 1974), a list of words is read aloud to the subject and then the subject is required to recall the list in consecutive trials. Reminders of words missed are given after each trial. This test generates measures of Storage (SRT-S; total words recalled) and Continuous Retrieval (SRT-CR; total words consistently retrieved without intertrial cueing). Subjects also performed the Logical Memory (LM; paragraph recall) and Visual Reproduction (VR; design recall through reproduction) subtests of the Wechsler Memory Scale (WMQ) (Wechsler 1945), with recall at $30 \mathrm{~min}$ after item presentation.

We selected four additional neuropsychological tests to sample diverse aspects of schizophrenic cognitive dysfunction. From the revised Wechsler Adult Intelligence Scale (WAIS-R) (Wechsler 1981) we selerted the Vorabulary (VB; definitions of words), Digit Symbol (D-SYM; transpose a string of digits into assigned symbols), and Digit Span (DSPAN; immediate recall of digits forward and backward) subtests. We chose the VB subscale because of its high correlation with veroal IQ and its relative resistance to intellectual decline that may affect other subtests (Lezak 1983). The D-SYM subtest assesses sustained attention, visuomotor coordination, and response speed, and it correlates highly with performance IQ on the WAIS-R test (Lezak 1983). The D-SPAN subtest assesses span of apprehension, working memory, and sustained attention (Lezak 1983). Together, these tests should reflect the poor attention and slowed reaction time demonstrated in schizophrenic patients (Levin et al 1989). We also included the Perseverative Error score from the the Wisconsin Card Sort Test (WCST-PE) (Heaton 1981), which indexes a different schizophrenic dysfunction in category formation and shifting (Weinberger et al 1986). 
Table 1. Rapid-Eye Movement Measures

\begin{tabular}{|c|c|}
\hline & Mean + SD \\
\hline REM latency (min) & $56.3 \pm 40.5$ \\
\hline \multicolumn{2}{|l|}{ Total REM } \\
\hline$\%$ of total sleep & $22.9 \pm 7.8$ \\
\hline & $65.3 \pm 25.6$ \\
\hline density & $1.01 \pm 0.35$ \\
\hline \multicolumn{2}{|l|}{ First-period REM } \\
\hline $\begin{array}{l}\text { min } \\
\text { density }\end{array}$ & $\begin{array}{l}19.2 \pm 14.7 \\
0.90 \pm 0.53\end{array}$ \\
\hline
\end{tabular}

$n=18$

\section{Statistical Analysis}

For statistical analysis, we transformed most of the sleep variables to more closely approximate normal distributions, selecting square-root transformations for REM latency and REM minutes and logarithmic transformations for the REM density measures. We also checked scattergrams and performed nonparametric, Spearman Rank correlations to ensure that outliers did not cause spurious correlation coefficients.

\section{Results}

Sleep EEG results for REM sleep are presented in Table 1. The findings were consistent with those from a larger sample that we have reported elsewhere (Tandon et al 1992), of which this sample represents a subset. The BPRS global severity score was $\mathbf{4 7 . 2} \pm$ 7.9 (mean $\pm S D$ ), and the mean total SANS score was $11.2 \pm 3.8$. For the neuropsychological test results, this sample had a full-scale IQ of $88.9 \pm 20.3$, a verbal IQ of $91.9 \pm 19.5$, a performance IQ of $86.8 \pm 19.2$ and a WMQ score of $93.7 \pm 21.9$. Results of the correlational analysis are presented in Table 2 and are summarized below.

Of the REM measures for the entire night, only REM density displayed a significant correlation and only with SRT-CR: poor performance on this memory task correlated with greater REM density. Our initial analysis of first-period REM time and density yielded six significant negative correlations with 16 cognitive measures, all opposite the predicted direction. Thus, poor cognitive performance predicted greater REM time and density.

To determine whether the first-period REM density measure influenced the correlation of iotal REM density with the SRT.CR score, we analyzed REM minutes and density for REM time occurring after the first REM period. Density for the later REM time did not correlate with SRT-CR, but minutes of REM time showed the predicted positive correlation with both SRT memory scores. Four of the six other cognitive tests also demonstrated statistical trends with REM time occurring after the first REM period. The Wisconsin Card Sort measure had a negative correlation, but this still represented a positive correlation with good performance because the score indicates errors made. Figure 1 shows the regression line for the SRT-CR scores plotted against first-period REM minutes and REM minutes after the first period.

Outliers did not unduly influence the results, except for a single outlier causing the VS scale to demonstrate a significant correlation with the first-period REM measures. 
Table 2. Correlation Matrix for REM and Neuropsychological Variables

\begin{tabular}{|c|c|c|c|c|c|c|c|c|}
\hline \multirow{2}{*}{$\begin{array}{l}\text { Neuropsychological } \\
\text { variables }\end{array}$} & \multirow{2}{*}{$\begin{array}{l}\text { REM latency } \\
\text { (min) }\end{array}$} & \multicolumn{3}{|c|}{ Total REM } & \multicolumn{2}{|c|}{ Ist-period REM } & \multicolumn{2}{|c|}{$\begin{array}{l}\text { Total minus 1st } \\
\text { period REM }\end{array}$} \\
\hline & & $\%$ of sleep & $\min$ & density & $\min$ & density & $\min$ & density \\
\hline \multicolumn{9}{|l|}{ Selertive reminding } \\
\hline SRT-S & -0.09 & 0.01 & 0.37 & -0.40 & -0.27 & $-0.48^{a}$ & $0.53^{a}$ & 0.21 \\
\hline SRT-CR & -0.18 & -0.26 & 0.27 & $-0.47^{a}$ & $-0.49^{a}$ & $-0.67^{b}$ & $0.57^{a}$ & 0.14 \\
\hline Logical Memory (LM) & -0.01 & -0.03 & 0.33 & -0.21 & -0.19 & -0.20 & $0.41^{c}$ & 0.10 \\
\hline Visual Reproduction (VS) & -0.32 & 0.06 & -0.12 & -0.03 & -0.24 & -0.10 & 0.24 & -0.29 \\
\hline Vocabulary (VB) & -0.10 & 0.02 & 0.21 & -0.09 & -0.39 & -0.29 & $0.44^{c}$ & 0.23 \\
\hline $\begin{array}{l}\text { Digit. Symbol Substitution } \\
\text { (D)-SYM) }\end{array}$ & -0.08 & -0.08 & 0.21 & -0.26 & -0.39 & $-0.56^{a}$ & $0.44^{c}$ & 0.10 \\
\hline Digit Span (D-SPAN) & -0.03 & -0.16 & 0.07 & -0.40 & $-0.53^{a}$ & $-0.54^{a}$ & 0.36 & 0.04 \\
\hline $\begin{array}{l}\text { Wisconsin Card Sort } \\
\text { Perseverative Error } \\
\text { (WCST-PE) }\end{array}$ & -0.09 & 0.23 & 0.27 & 0.00 & 0.17 & 0.13 & $-0.48^{c}$ & -0.09 \\
\hline
\end{tabular}

Pearson Product Moments calculated for 18 subjects, except for WCST-PE $(n=15)$.

ap $<0.05$

$b_{p}<0.005$

op $<0.1$

Analysis excludes single outlier.

This correlation fell to levels of nonsignificance when the outlier was removed. Nonparametric tests yielded similar results for all test.

Measures of symptom severity (BPRS, SANS) and age did not correlate with any of the neuropsychological test scores, with REM minutes, or with REM density for any time of the night. As we have previously found that withdrawal of psychotropic medication for less than 4 wks before PSG recording can cause elevated REM activity in the first period (Tandon et al 1992), we recalculated correlations for the strongest associations, excluding from our sample the five patients who were withdrawn from neuroleptic medication more than 2 but less than 4 wks before the sleep EEG study. The correlations, though weaker, retained significance at the $p=0.05$ level or deinonstrated a statistical trend. For first-period density, they were $-0.48(\mathrm{df}=12, p=0.09)$ with SRT-CR and -0.55 (df $=12, p=0.05$ ) with D-SPAN.

\section{Discussion}

Our results suggest two opposing correlations of REM sleep with cognition and memory. REM time exhibited the predicted positive correlation with cognitive function, but only for REM time after the first REM period. First-period REM time and REM density, sometimes referred to as "phasic REM," correlated negatively with several measures of cognition. Our failure to find a significant association for total REM time with cognition can be seen as the confluence of our expected finding with an unexpected finding for REM time in the first period. Opposing correlations negated each other when we analyzed REM time for the entire night. These results suggest that depending on when it occurs during sleep, REM sleep has very different associations with intellectual function.

For REM time after the first period, our results confirm previous reports in the literature. Though the SRT test of memory demonstrated the only significant correlation with REM 


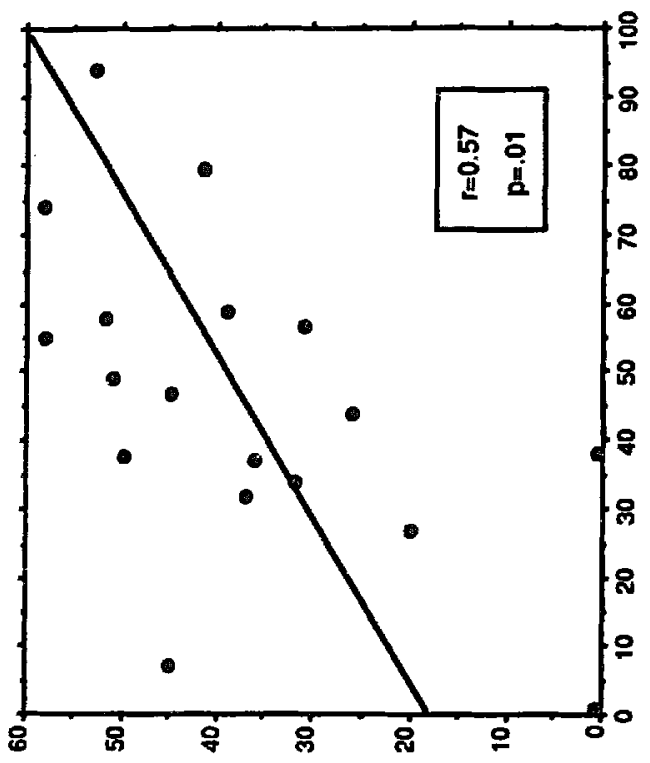

急

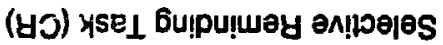

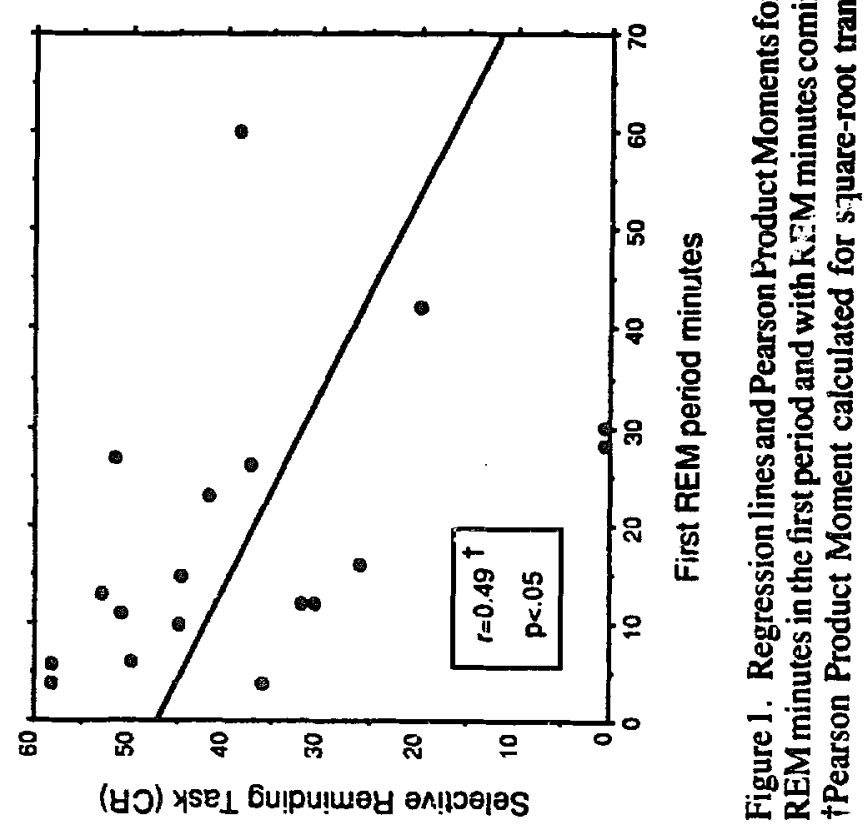


time, the statistical trends obtained from the other psychometric tests (VB, D-SYM, and WCST-PE) suggested a positive association with more global cognitive function. The consistent finding of REM time connected with intellectual function in groups of elderly normal subjects (Feinberg et al 1967; Kahn and Fisher 1969; Prinz 1977), elderly demented patients (Feinberg et al 1967), abstinent alcoholics (Benson et al 1978), and developmentally disabled persons (Feinberg 1968; Castaldo and Krynicki 1974) argues for an association between REM sleep and information processing. This has important implications for the study of REM sleep physiology. These findings are consistent with the hypothesis of Crick and Mitchison (1983) that REM sleep provides a "reverse learning" input to cortical networks that increases their information processing efficiency.

Why should the first REM period differ from REM occurring later in the night? Investigations of normal sleep physiology have identified a greater sensitivity of the first REM period to pharmacologic manipulations (Nicholson et al 1989). Experiments that have manipulated the amount of REM sleep to look for an effect on recent memory consolidation have suggested a differential role for REM sleep, depending on the time of occurrence. Studies of REM sleep deprivation in animals have suggested that a "critical window" for memory consolidation exists in the early part of the night (Horne and McGrath 1984; Smith 1985). Studies of sleep and learning in humans have also identified the first part of the night as crucial for the consolidation of recent information (Ekstrand et al 1977; Fowler et al 1973).

These more explicit tests of a REM-memory consolidation hypothesis differ significantly from our correlational analysis, which did not impose any "information load" with the testing procedures. In fact, one or two days usually separated the testing from polysomnography. Differences in polysomnographic methods require caution in making comparisons between various studies. For instance, animal polysomnography usually does not measure eye movements, and thus, does not examine REM density. Although we found parallel correlations for first-period REM density and duration, these are not identicul neurophysiological processes. With these caveats, these tests of a REM-memory consolidation hypothesis do suggest a state-dependent role for REM sleep occurring in the early part of the night.

A state-dependent role for early REM sleep, combined with a correlation opposite that expected, suggests several possibilities. Early REM sleep, through some abnormality, may have caused poor cognitive performance. However, first-period REM density and duration in schizophrenics have been found not to differ from normal subjects when medication-withdrawal effects are excluded (Keshavan et al 1990; Tandon et al 1992), so such an abnormality would have to be fairly subtle. A third process, which would influence REM and cognition separately, could have caused the observed association. The stress experienced in the course of a psychotic break and hospitalization is an obvious candidate. Although we do not find an association for REM time and REM density with symptom severity that might be expected in this case, we cannot rule out stress or some other third mechanism causing a coincidental association.

One other possibility is that the early REM sleep negatively associated with cognitive function may represent a compensatory mechanism. Tandon and Greden (1989) have suggested that when hypothesized doparninergic hyperactivity occurs during a schizophrenic episode, symptoms are modulated by a compensatory increase in cholinergic activity. The data of the current study can be explained by such a model. Dopaminergic hyperactivity has been linked with poor cognitive function in schizophrenics (Matthysse 1978), and dopamine blockade generally improves the cognitive performance of schizo- 
phrenics (King 1990). In other studies in our Schizophrenia Program, we have found that when challenged with biperiden (a muscarinic antagonist), the first-period REM density of schizophrenic patients-at baseline no different than normal controls-exhibits a greater decrease (Tandon et al 1991). One interpretation of this greater sensitivity of phasic REM activity to muscarinic blockade is greater muscarinic activity stimulating REM-generating neurons in schizophrenics.

Early phasic REM, driven by muscarinic neurons (Sitaram et al 1978), may be responding to those patients with very disordered cognition with a compensatory increase. Questions remain however, about what specific cognitive function might elicit the compensatory response. Though the associations of first-period REM density and duration with cognitive function were more specific than those with later REM time, in the current study we cannot reliably identify any specific cognitive function (such as memory or sustained attention) beyond noting that the D-SPAN, D-SYM, and SRT tasks appear to require this function.

Longitudinal assessments of sleep and neuropsychological measures could provide important information regerding these hypotheses. For instance, if the phasic REM activity of the first period plays a compensatory role, then we might expect a positive correlation between this measure and improvement on cognitive scores. Also, for those patients who have poor cognition and do not show increases in first-period REM time or density, we would expect less improvement over time on neuropsychological test scores. We are currently collecting longitudinal neuropsychological data in order to address this question.

Our findings suggest further investigation and the need for replication, particularly with the relatively small number of subjects and the risk of a typ: I error from the many statistical tests performed without adjustment. In addition to questions about the general validity of this finding for the first-period REM sleep, one would also want to know if this relationship held for other psychiatric diagnoses. If this finding does have validity, then it may have important implications for understanding normal sleep physiology and the pathophysiology of schizophrenia.

This paper was presented at the 47th Annual Meeting of the Society of Biological Psychiatry, Washington DC, 1992. The authors would like to thank John Greden and Cynthia Pomerleau for helpful suggestions and editorial assistance.

\section{References}

Andreasen NC (1983): The scale for the Assessment of Negative Symptoms. Iowa City: University of Iowa Press.

American Psychiatric Association (1987): Diagnostic and Statistical Manual of Mental Disorders, Third Edition, Revised (DSM-III-R). Washington, D.C.: American Psychiatric Association.

Benson K, Cohen M, Zarcone V (1978): REM sleep time and digit span impairment in alcoholics. J Stud Alcohol 39:1488-1498.

Buschke H, Fuld PA (1974): Evaluating storage, retention, and retrieval in disordereu axisury and learning. Neurology 11:1019-1025.

Castaldo V, Krynicki V (1974): Sleep and eye movement patterns in two groups of retardates. Biol Psychiatry 9:231-244.

Crick F, Mitchison G (1983): The function of dream sleep. Nature 304:111-114.

Dujardin K, Guerrien A Leconte P (1990): Sleep, brain activation and cognition. Physiol Behav 47:1271-1278.

Endicott J, Spitzer RL (1978): A diagnostic interview: The schedule for affective disorders and schizophrenia (SADS). Arch Gen Psychiatry 35:837-853. 
Ekstrand BR, Barrett TR, Est JN, Maier WG (1977): The effect of sleep on human long-term memory. In Drucker-Coline, McGaugh JL (eds): Neurobiology of Sleep and Memory. New York: Academic Press, pp 419-438.

Feinberg I (1968): Eye movement activity during sleep and intellectual function in mental retardation. Science 159:1256.

Feinberg I, Koresko RL, Heller N (1967): EEG sleep patterns as a function of normal and pathological aging in man. J Psychiat Res 5:107-144.

Fowler MJ, Sullivan MJ, Ekstrand BR (1973): Sleep and memory. Science 179:302-304.

Greenberg R, Pearlman C, Schwartz WR Grossman HY (1983): Memory, emotion and REM sleep. $J$ Abnorm Psychol 92:378-381.

Heaton RK (1981): A Manual for The Wisconsin Card Sorting Test. Odessa, FL: Psychological Assessment Resources.

Horne JA, McGrath MJ (1984): The consolidation hypothesis for REM sleep function: Stress and other confounding factors - a review. Biol Psychol 18:165-184.

Kahn E, Fisher C (1969): Some correlates of rapid eye movement sleep in the normal aged male. J Nerv Ment Dis 148:495-505.

Keshavan MS, Reynolds CF Kupfer DJ (1990): Electroencephalographic sleep in schizophrenia: A critical review. Compr Psychiatry 30:34-47.

King DJ (1990): The effect of neuroleptics on cognitive and psychomotor function. Br J Psychiatry 157:799-811.

Levin S, Yurgelun-Todd D Craft S (1989): Contributions of clinical neuropsychology to the study of schizophrenia. J Abnorm Psychol 98:34 1-356.

Lezak MD (1983): Neuropsychological Assessment. New York: Oxford University Press.

Matthysse S (1978): A theory of the relation between dopamine and attention. J Psychiat Res 14:241-248.

Nicholson AN, Belyavin AJ, Pascoe PA (1989): Modulation of rapid eye movement sleep in humans by drugs that modify monoaminergic and purinergic transmission. Neuropsychopharmacology 2:131-143.

Overall JE, Gorham DR (1962): Brief psychiatric rating scale. Psychol Reports 10:799-812.

Prinz PN (1977): Sleep patterns in the healthy aged: Relationship with intellectual function. $J$ Gerontol 32:179-186.

Rechtschaffen A, Kales A (1968): A Manual of Standardized Terminology, Techniques, and Scoring System for Sleep Stages in Human Subjects. Bethesda, MD: National Institutes of Nervous Disease and Blindness.

Saykin AJ, Gur RC, Gur RE, et al (1991): Neuropsychological function in schizophrenia. Arch Gen Psychiatry 48:618-624.

Scrima L (1982): Isolated REM sleep facilitates recall of complex associative information Psychophysiology 19:252-259.

Sitaram N, Moore AM Gillin JC (1978): Induction and resetting of REM sleep rhythm in normal man by arecholine: blockade by scopolamine. Sleep 1:83-90.

Smith C (1985): Sleep states and learning: a review of the animal literature. Neurosci Biobehav Res 9:157-168.

Spitzer RL, Endicotl J, Robins E (1978): Research diagnostic criteria (RDC): Rationale and reliability. Arch Gen Psychiatry 35:773-782.

Tandon R, Greden JF (1989): Cholinergic hyperactivity and negative schizophrenic symptoms: A model of cholinergic/dopaminergic interactions in schizophrenia. Arch Gen Psychiatry 46:745753.

Tandon R, Shipley JE Taylor S (1991): Muscarinic hyperactivity in schizophrenia: Effect of anticholinergic biperiden on REM sleep in schizophrenic patients ard normals. Biol Psychiatry 29:72A. 
Tandon R, Shipley JE, Taylor SF, et al (1992): Electroencephalographic sleep abnormalities in schizophrenia: Relationship to positive/negative symptoms and prior neuroleptic treatment. Arch Gen Psychiatry 49:185-194.

Taylor MA Abrams R (1984): Cognitive impairment in schizophrenia. Am J Psychiatry 141:196201.

Wechsler D (1945): A standardized memory scale for clinical use. J Psychology 19:87-95.

Wechsler D (1981): WAIS-R manual. New York: Psychological Corporation.

Weinberger DR, Berman KF Zec RF (1986): Physiologic dysfunction of dorsolateral prefrontal cortex in schizophrenia. Arch Gen Psychiatry 43:114-124. 